\title{
Hemocultivos en recién nacidos: optimizando la toma de muestra y su rendimiento
}

\author{
Giannina Izquierdo, Patricia García, Marta Aravena, Luis Delpiano, Alejandra Reyes, \\ Fernanda Cofré, Mariluz Hernández, Alejandra Sandoval y Yenis Labraña
}

\section{Blood cultures in newborns: optimizing sample collection and performance}

The request of blood cultures in medical care is frequent, especially in Neonatal Units, where it is performed routinely in case of suspected early or late sepsis. The purpose of this document is to standardize the sampling technique in order to increase its performance and establish criteria to interpret a positive blood culture.

Key words: Blood cultures, neonates, sepsis.

Palabras clave: Hemocultivos, recién nacidos, sepsis.

\section{Introducción}

$$
\mathrm{L}
$$

a sepsis es una de las patologías más importantes en recién nacidos $(\mathrm{RN})$ hospitalizados en Unidades de Neonatología considerando su frecuencia y alta morbimortalidad. El diagnóstico clínico es difícil debido a que presenta una manifestación inespecífica y los métodos de detección utilizados no cuentan con un rendimiento óptimo. Por esto, el equipo médico habitualmente indica un tratamiento antimicrobiano empírico y precoz ante su sospecha, lo que se traduce en un uso excesivo de antimicrobianos.

A pesar del advenimiento de técnicas moleculares para la detección de bacterias y virus en sangre, el estándar de oro para el diagnóstico de sepsis neonatal sigue siendo el hemocultivo $(\mathrm{HC})^{1,2}$. Este método tiene la ventaja de poseer una alta especificidad, permite la identificación del microorganismo y realizar el estudio de susceptibilidad de éste. Sin embargo, presenta desventajas como la latencia en el resultado, que alcanza entre 24 a $72 \mathrm{~h}$, o la difícil interpretación si se aísla Staphylococcus coagulasa-negativa ( $\mathrm{SCN})$. SCN es el microorganismo más frecuentemente aislado en sepsis neonatal tardía, con incidencias que fluctúan entre 29 y $50 \%^{3-5}$, pero a su vez, también es el más frecuente en muestras contaminadas, alcanzando una tasa de falsos positivos de hasta $17 \%$ en RN bajo 12 semanas de vida, lo que se traduce en un gran desafío para el médico tratante, quien tiene que distinguir entre sepsis y contaminación ${ }^{1,6-8}$.

Sin lugar a dudas, la principal desventaja de esta técnica microbiológica es su baja sensibilidad (hasta $60 \%$ de los RN con sepsis clínica tienen HC negativos) y esto se puede deber a varias razones:

- volumen insuficiente de sangre extraída,
- baja carga de microorganismos en bacteriemias en este tipo de paciente (bacteriemia de bajo nivel definidas como $<4 \mathrm{ufc} / \mathrm{ml}$ ),

- presencia de microorganismos fastidiosos o anaerobios $\mathrm{y}$

- la obtención de la muestra en el RN con posterioridad a la administración de antimicrobiano en la madre?.

Existe muy poca información en la literatura médica, en cuanto al volumen necesario de sangre a tomar, incluso recomendaciones contradictorias en relación al porcentaje de sangre de la volemia ${ }^{10}$, número de frascos, sitio y momento de la extracción de la muestra de $\mathrm{HC}$ en Neonatología. Por esta razón, el Comité de Infecciones Neonatales de la Sociedad Chilena de Infectología, se propuso profundizar en el tema para intentar aclarar al máximo las interrogantes y sugerir una pauta de estudio en este tipo de paciente.

\section{Clasificación de los hemocultivos}

El HC o cultivo microbiológico de la sangre se puede clasificar según el tipo de paciente (neonatal, pediátrico, adulto), el tipo de toma de muestra (centrales o periféricos); tipo de microorganismo (bacterias aerobias, anaerobias, fastidiosas o micobacterias) y según la metodología de los distintos sistemas de HC. Existen sistemas convencionales (manuales), sistemas semi-automatizados (lisis- centrifugación) o sistemas automatizados como BACTEC o BacT/Alert, entre otros.

Los sistemas manuales tienen la desventaja en relación al automatizado, de tener un mayor riesgo de contaminación al manipular las botellas para realizar los
Comité Consultivo de Infecciones Neonatales. Sociedad Chilena de Infectología.

Hospital Exequiel González Cortes (GI); Departamento de Laboratorios Clínicos. Escuela de Medicina. Pontificia Universidad Católica de Chile (PG); Hospital Sótero del Río (MA); Hospital San Borja Arriarán (LD); Hospital Félix Bulnes Cerda (AR); Hospital Roberto del Río (FC); Hospital Luis Tisné (MH); Hospital Carlos Van Buren (AS) y Hospital San Juan de $\operatorname{Dios}(\mathrm{YL})$.

Recibido: 11 de marzo de 2018

Correspondencia a: Giannina Izquierdo Copiz gianninai@yahoo.es 
procedimientos de tinción, son más laboriosos ya que requieren de un sub-cultivo para detectar el desarrollo bacteriano/fúngico, lo que origina resultados en plazos más largos.

Los semi-automatizados o de lisis centrifugación también son laboriosos, requieren manipulación, son de alto costo y a pesar que permiten la recuperación de bacterias intracelulares, no han mostrado mejor sensibilidad que los sistemas automatizados ${ }^{11-13}$.

Los sistemas automatizados consisten básicamente en botellas con diversos medios de cultivo (aeróbicos, anaeróbicos, hongos, micobacterias y con resinas que captan antimicrobianos) que se incuban en equipos que agitan constantemente las muestras y poseen métodos de detección de productos del metabolismo bacteriano $\left(\mathrm{CO}_{2}\right)$. Mediante sistemas radiométricos, colorimétricos o fluorométricos continuos, se determina un umbral de producción de $\mathrm{CO}_{2}$ considerado como positividad del hemocultivo. Las botellas se retiran del equipo y se les realiza una tinción de Gram que permite un informe preliminar y precoz respecto de la forma, afinidad tintorial y disposición de los microrganismos en desarrollo. Esta técnica ha permitido aumentar la velocidad de detección del agente en desarrollo, disminuir el tiempo de respuesta y aumentar la sensibilidad de los HC. Por otra parte, ha disminuido la carga de trabajo en el laboratorio, la contaminación cruzada y ha aumentado el espectro de microorganismos detectados. Este método permite mejorar la capacidad diagnóstica en bacteriemias y por ende, un uso más racional de la terapia antimicrobiana ${ }^{14}$.

\section{Volumen de sangre}

Múltiples estudios han confirmado que en adultos la variable más crítica para optimizar el rendimiento del $\mathrm{HC}$ es el volumen de sangre. Hasta hace un tiempo, se pensaba que los pacientes pediátricos que cursaban con bacteriemias tenían recuentos bacterianos altos, esto basado en las bacteriemias neonatales causadas por Escherichia coli ${ }^{15}$ por lo que eran aceptados volúmenes pequeños, inferiores a $1 \mathrm{ml}$. Sin embargo, datos de Schelonka y cols. ${ }^{16}$, demostraron que volúmenes de 0,5 $\mathrm{ml}$ no eran capaces de detectar bacteriemias de bajo nivel definidas como aquellas menores a $4 \mathrm{ufc} / \mathrm{ml}$. Hoy se sabe que alrededor de $25 \%$ de las bacteriemias en lactantes tienen un recuento menor a $4 \mathrm{ufc} / \mathrm{ml}$ y dos tercios de los lactantes bajo dos meses de edad tienen recuentos menores de $10 \mathrm{ufc} / \mathrm{ml}^{17}$. Si los sistemas automatizados pueden detectar hasta $1 \mathrm{ufc} / \mathrm{ml}$, entonces hay que tomar al menos $1 \mathrm{ml}$ de sangre e inocular una botella de hemocultivos aeróbica pediátrica. En conclusión, si se toma un volumen adecuado de muestra, se podría aumentar la positividad al doble ${ }^{16}$.

\section{Relación sangre/medio de cultivo}

Además del volumen de sangre, la actividad bactericida de ésta disminuye en sí el rendimiento de los HC. Es importante tener presente que la sangre tiene un poder bactericida relacionado a la inmunidad innata (complemento, actividad fagocítica de los leucocitos y lisozimas) sumado al efecto que pudiese tener el uso de antimicrobianos en la madre, previo al parto.

La recomendación es mantener una relación de al menos 1:5 entre la muestra y el volumen de medio de cultivo; esta dilución permite neutralizar las propiedades bactericidas de la sangre y de los agentes antibacterianos que puedan estar presentes en la muestra. En el caso de los sistemas automatizados pediátricos, las botellas están diseñadas para mantener la relación 1:5 con volúmenes menores de sangre ${ }^{11}$.

\section{Elección del medio de cultivo}

El cultivo en medio anaeróbico además del aeróbico no aumenta el rendimiento de los $\mathrm{HC}$ en la población pediátrica en general ${ }^{18}$. Podría existir un mayor riesgo de bacteriemia por anaerobios en los pacientes con sepsis de foco abdominal u oral, con mordeduras de animales, inmunocomprometidos o con úlceras de decúbito ${ }^{19,20}$ por lo que se podrían considerar en este grupo.

El cultivo en botella anaeróbica en RN está recomendado para situaciones especiales como, antecedentes de rotura prolongada de membranas (RPM), corioamnionitis materna, sepsis de origen abdominal (enterocolitis necrosante) o sepsis nosocomial tras cirugía abdominal ${ }^{21}$. Un estudio de la década de los noventa reportó incidencias variables de bacteriemia por anaerobios en neonatos entre 1,8 y $12,5 \%$ con los factores predisponentes previamente descritos, siendo más frecuente el aislamiento de Bacteroides $\mathrm{spp}^{22}$. Por otra parte, un estudio reciente, retrospectivo, describe que $33,9 \%$ de las bacteriemias pesquisadas en pacientes bajo 28 días de vida se aíslan sólo en frascos de HC anaeróbicos y no en los aeróbicos, por lo que tendrían mayor rendimiento en la pesquisa de microorganismos no necesariamente anaerobios facultativos. El único anaerobio facultativo estricto encontrado fue Clostridium symbiosum en un $\mathrm{HC}^{23}$.

Yaacobi y cols., evaluaron, mediante un estudio prospectivo, el rendimiento de los $\mathrm{HC}$ utilizando dos botellas con $0,5 \mathrm{ml}$ de sangre (aeróbico y anaeróbico) comparado con inocular $1 \mathrm{ml}$ de sangre en una botella de $\mathrm{HC}$ aeróbico en RN con sospecha de sepsis. El rendimiento global fue de $10,2 \%$ y mayor en el grupo de dos muestras de $0,5 \mathrm{ml}$, pero los microrganismos aislados en el frasco anaeróbico fueron predominantemente agentes aeróbicos, lo que sugiere que el rendimiento aumentaría, pero no queda 
claro si fue por la toma de dos frascos de $\mathrm{HC}$ o por el medio anaeróbico ${ }^{24}$.

A la fecha, no hay estudios comparativos entre cultivos aeróbicos y anaeróbicos con poder suficiente para recomendar el uso de $\mathrm{HC}$ anaeróbicos en todos los $\mathrm{RN}$, pero se sugiere considerarlo en aquellos con factores de riesgo para infección por anaerobios, sobre todo en aquellos con antecedentes de RPM, corioamnionitis y patología abdominal quirúrgica. El volumen mínimo a sembrar debe ser también de $1 \mathrm{ml}$.

\section{Número de hemocultivos}

Existe escasa información sobre el número de $\mathrm{HC}$ necesarios a tomar en RN y sobre todo en RN de muy bajo peso de nacimiento (definidos como aquellos bajo $1.500 \mathrm{~g}$ ), donde el principal riesgo de la extracción de sangre es la anemia secundaria con necesidad de transfusiones frecuentes. A su vez, en toda edad se recomienda la toma de $\mathrm{HC}$ de al menos dos sitios distintos.

Como Comité recomendamos tomar al menos dos $\mathrm{HC}$ de $1 \mathrm{ml}$ cada uno extraídos de sitios diferentes. Esto permite diferenciar una contaminación de la real bacteriemia en el caso de aislar SCN. La presencia de SCN en dos botellas de HC tomadas de sitios distintos permite diferenciar la contaminación con infección. En el caso de no poder tomar dos $\mathrm{HC}$ por las condiciones del paciente, se puede tomar un hemocultivo, pero con al menos $1 \mathrm{ml}$ de sangre ${ }^{25}$.

Frente a la sospecha de infección de torrente sanguíneo asociada a catéter venoso central (ITS/CVC), importante causa de infección asociada a atención de salud en UCI neonatal, el diagnóstico se hace más complejo. Clásicamente se ha definido ITS/CVC como:

- Presencia de manifestaciones clínicas sugerentes o evidentes de ITS/CVC con un cultivo de punta de CVC con un recuento significativo $(>15 \mathrm{ufc} / \mathrm{ml})$ asociado a la presencia de un $\mathrm{HC}$ periférico positivo al mismo microorganismo.

- Un HC cuantitativo con un mismo microorganismo obtenido desde el CVC y punción periférica con una relación $\geq 3: 1$, respectivamente.

- Un tiempo diferencial de positividad de $\geq 2 \mathrm{~h}$ (se identifica $2 \mathrm{~h}$ antes en el CVC que en el HC periférico) en $\mathrm{HC}$ automatizados.

Para realizar este diagnóstico se necesita de la recolección de más de una muestra de $\mathrm{HC}$ en la mayoría de los casos.

Según un estudio realizado en nuestro país por Acuña y cols., quienes compararon el rendimiento de $\mathrm{HC}$ cuantitativo y tiempo diferencial de positividad, se pudo demostrar que este último presenta una mayor sensibilidad
(24 vs 75\%) para el diagnóstico de ITS/CVC en niños, comprobando que este método representa una mejor alternativa ${ }^{26}$.

En el caso de CVC con doble lumen, se ha intentado evaluar el rol de métodos alternativos como $\mathrm{HC}$ de ambos lúmenes y ver el diferencial en cuanto al recuento o tiempo; sin embargo, este procedimiento tiene una baja sensibilidad y bajo valor predictor positivo, por lo que aún no está validado ${ }^{27,28}$.

En algunos centros extranjeros se ha implementado la modalidad de tomar $0,5 \mathrm{ml}$ de cada lumen del CVC e inocular en un solo frasco de $\mathrm{HC}$ aeróbico pediátrico para así optimizar el diagnóstico, practica no validada aún en ensayos clínicos, pero que pareciera ser adecuado en el caso de RN de muy bajo peso de nacimiento, donde la importancia radica en el diagnóstico de la infección y no cuál es el lumen infectado (información no publicada).

La principal dificultad en el diagnóstico en este grupo de pacientes, nuevamente es el volumen de sangre. Se ha visto que la adherencia a la recomendación de obtener $\mathrm{HC}$ pareados es de sólo $58 \%{ }^{29}$, por lo que el médico tratante debe trabajar sobre un diagnóstico presuntivo, lo que implica prolongación innecesaria de antibioterapia y retraso en el diagnóstico de otra etiología.

\section{Sitio de obtención de la muestra}

\section{Vasos umbilicales}

Se puede tomar hemocultivos tanto de la vena como de la arteria umbilical.

La vena tiene mayor probabilidad de contaminación que la arteria umbilical, riesgo que disminuiría si al momento del parto este vaso es doblemente clampeado y preparado adecuadamente ${ }^{30-32}$.

Existe antigua evidencia que la utilización de sangre de arteria umbilical para la toma de $\mathrm{HC}$ otorga buenos resultados. Pourcyrous M y cols., realizaron un estudio prospectivo en 282 RN con 318 hemocultivos pareados: sangre periférica vs catéter de arteria umbilical, describiendo una tasa de contaminación: $1,3 \%$ en vena periférica y de $0,9 \%$ en sangre de arteria umbilical. Hubo 13 pares de $\mathrm{HC}$ concordantes (mismo microorganismo) y sólo 2 pares de $\mathrm{HC}$ discordantes. Esto plantea que en $\mathrm{RN}$ hospitalizados la obtención de muestra para $\mathrm{HC}$ desde catéter de arteria umbilical recién instalado puede ser una alternativa $^{30}$.

\section{Hemocultivos de cordón}

La obtención de una muestra de hemocultivos a través de cordón umbilical permite tomar un volumen de sangre significativamente mayor ya que esto no produciría anemia en el RN. La sangre del cordón umbilical pasa a través de la membrana del trofoblasto, la que se 
define como una membrana semipermeable, que separa la circulación materna y fetal. Por lo tanto, la sangre en el cordón umbilical es sangre fetal y es idéntica a la sangre del feto.

Así el volumen es fácilmente accesible, permitiendo un aumento de la sensibilidad de los HC. La técnica de recolección de $\mathrm{HC}$ de cordón es crítica, pues existe alto riesgo de contaminación post parto vaginal ${ }^{33,34}$.

Los principales beneficios que ofrece esta técnica son:

- Obtención inmediata de la muestra, sin retrasar el diagnóstico y permitiendo rápidamente el inicio de antimicrobianos empíricos en niños con sospecha de sepsis precoz.

- Obtención de volúmenes adecuados.

- Una menor tasa de transfusiones en el RN, al reducir los volúmenes extraídos, especialmente en $\mathrm{RN}$ de pretérmino.

- Es un método no invasor, no traumático e indoloro para el RN.

- Implica menos tiempo y menor costo ${ }^{34,35}$.

Meena y cols., analizaron la utilidad de los $\mathrm{HC}$ de cordón en $40 \mathrm{RN}$ con factores de riesgo para sepsis, es decir, prematuridad, rotura de membranas de $>24 \mathrm{~h}$, líquido amniótico de mal olor, fiebre materna, tactos vaginales frecuentes, asfixia neonatal y peso de nacimiento $<2.500$ g. La técnica utilizada en este estudio fue la siguiente: una vez que nació el RN, el cordón umbilical fue clampeado en el extremo placentario y en el extremo umbilical, cortando el cordón entre los clamps. Se realizó una desinfección del extremo placentario con alcohol $70^{\circ}$ y se recolectó una muestra de sangre de $2 \mathrm{ml}$, que fueron sembrados en una botella de $\mathrm{HC}$ aeróbico. La sensibilidad de este método en este estudio fue de $100 \%$ con una especificidad de $94,9 \%$ en comparación con los HC tomados en vena periférica ${ }^{34}$. Por otra parte, existen otros reportes que describen una sensibilidad un poco menor, cercanas a $80 \%$, con especificidad de $91,4 \%$ comparadas con $\mathrm{HC}$ tomados por vía periférica ${ }^{36}$.

La principal limitación de la toma de muestra del cordón umbilical es la contaminación y a pesar de que hay métodos para disminuir este riesgo, hasta el momento no hay recomendaciones que incluyan meta-análisis o estudios caso-controles que lo avalen ${ }^{37}$. Se deben realizar más experiencias al respecto debido a que impresiona ser una técnica que aumenta la tasa de aislamiento del microrganismo sin mayor morbilidad para el $\mathrm{RN}$ con sospecha de sepsis precoz.

\section{Antiséptico a emplear}

Además del aseo de la piel, retirando materia orgánica, el uso de antiséptico disminuye la probabilidad de contaminación de las muestras para HC. En las unidades neonatales, habitualmente se dispone de alcohol y clorhexidina como antisépticos para la práctica clínica, y muy ocasionalmente povidona yodada. Estos antisépticos difieren en varios aspectos y poseen ventajas o desventajas que están ampliamente expuestas en un documento de nuestra Sociedad Científica ${ }^{38}$.

Un estudio comparativo evaluó eficacia de clorhexidina $1 \%$ versus povidona iodada al $10 \%$ en evitar contaminación en la toma de muestras venosas de hemocultivos en UCI neonatal, siendo menor en el grupo de clorhexidina (0 vs $2,9 \%, p=0,026)^{39}$.

Debemos recordar que clorhexidina posee alta acción germicida pero la de base acuosa requiere de tres minutos antes de iniciar el procedimiento y la de base alcohólica $(0,5$ a $2 \%)$ requiere al menos 30 segundos; a diferencia del alcohol $70 \%$ cuyo efecto bactericida se inicia en 15 segundos. Para ambos antisépticos debe existir precaución respecto de emplear altas cantidades pues resultan irritantes para la piel en particular del recién nacido de muy bajo peso, debiendo seleccionarse el que este normado en cada institución.

\section{Tiempo de positividad de los hemocultivos}

El determinar el tiempo de positividad de los $\mathrm{HC}$ en cada unidad es fundamental para conocer el tiempo en el que se espera tener un $\mathrm{HC}$ positivo en un paciente que se sospecha sepsis y después de cuántas horas poder suspender los antimicrobianos iniciados en forma empírica, con tranquilidad. Por otra parte, permite diferenciar las infecciones de la contaminación.

Recientemente se describió que el tiempo promedio de positividad de $\mathrm{HC}$ tomados en $826 \mathrm{RN}$ con sospecha de sepsis precoz admitidos en UCIN fue de 21,1 h. Ellos concluyen que no se requerirían más de tres días de incubación al usar el sistema BacT/ALERT 3D $60^{40}$.

\section{Interpretación del aislamiento de Staphylococcus coagulasa negativa}

Un aspecto relevante en la interpretación de $\mathrm{HC}$ y dado que $\mathrm{SCN}$ es uno de los principales agentes aislados, como Comité Consultivo proponemos adherir el esquema presentado en la Figura 1, a fin evitar falsas interpretaciones y mal uso de antimicrobianos ${ }^{37}$.

Finalmente, debido a las numerosas dificultades en la toma e interpretación de los hemocultivos, examen solicitado frecuentemente en las unidades neonatales, dejamos una tabla resumen de las recomendaciones para la obtención de HC en el RN (Tabla1). 


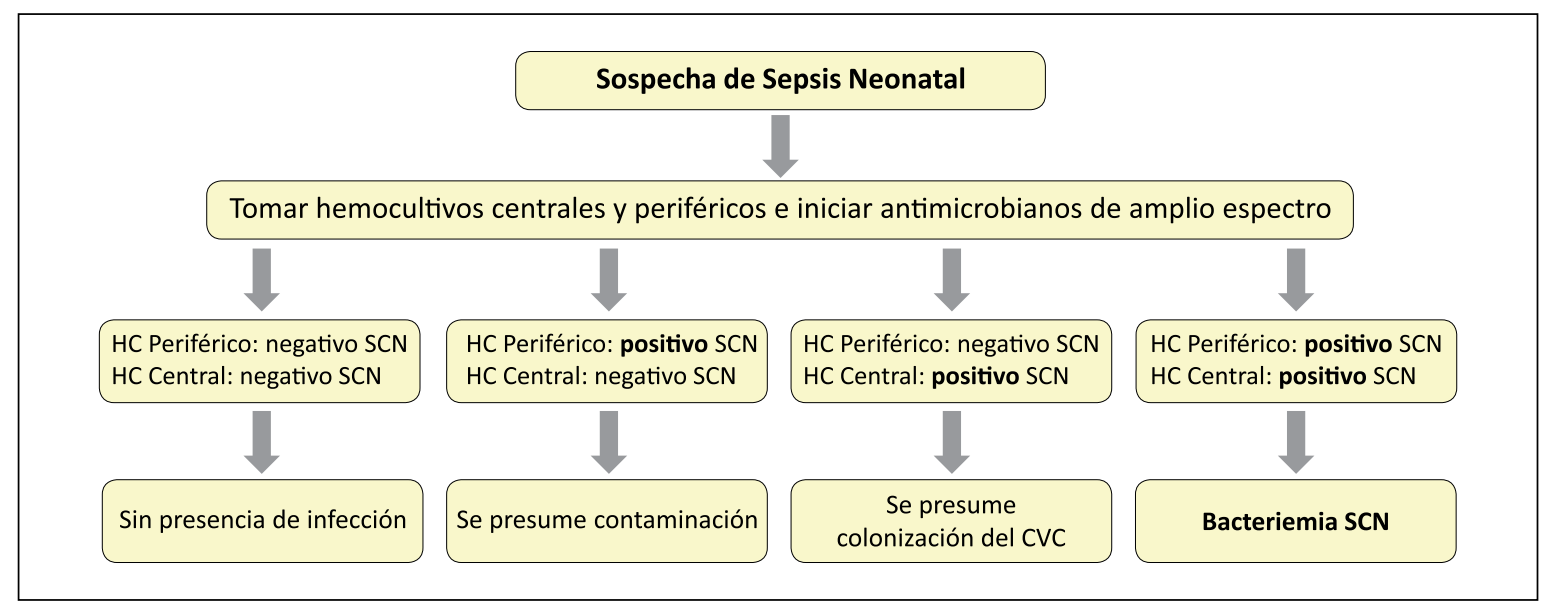

Figura 1. Algoritmo para interpretar hemocultivos positivos para Staphylococcus coagulasa negativo (SCN). Tomado de Polin R, Denson S, Brady M. Epidemiology and diagnosis of health care-associated infections in the NICU. Pediatrics 2012;129:e1104-9.

Tabla 1. Resumen de recomendaciones para a obtención de hemocultivos en recién nacidos.

Comité Consultivo de Infecciones Neonatales - Sociedad Chilena de Infectología.

1. Tomar hemocultivos (HC) siempre frente a la sospecha de sepsis

2. Tomar dos $\mathrm{HC}$ de diferentes sitios, con un volumen mínimo de $1 \mathrm{ml}$

3. Si el paciente está con vía venosa central intentar tomar un HC CVC (1 ml) y un HC periférico (1 ml)

4. Si el paciente tiene un CVC de más de un lumen, en RN prematuros extremos, se puede tomar 0,5 $\mathrm{ml}$ de cada lumen y sembrarlo en la misma botella de $\mathrm{HC}$ con el objetivo de aumentar del rendimiento, sin tener que extraer mayor cantidad de volumen

5. Considerar el uso de HC anaeróbicos en pacientes con factores de riesgo: rotura prematura de membranas, antecedentes de corioamnionitis materna y sepsis de foco abdominal, según la disponibilidad de cada centro. Volumen mínimo $1 \mathrm{ml}$

6. Considerar la toma de HC de arteria umbilical en catéteres recién instalados. Los HC de cordón tienen mayor tasa de contaminación, pero con alta capacidad de aislamiento del microorganismo Se deben realizar más experiencias al respecto

7. Normar la técnica de toma de HC en cada unidad Considerar tiempo de acción de antisépticos para evitar contaminación de las muestras

8. Parece seguro suspender antimicrobianos con hemocultivo negativos a las $48 \mathrm{~h}$ de incubación, en ausencia de evidencia clínica de infección bacteriana

\section{Resumen}

La solicitud de hemocultivos en la atención médica es frecuente, especialmente en las Unidades de Neonatología, donde se realiza en forma rutinaria frente a la sospecha de sepsis precoz o tardía. Este documento tiene como objetivo estandarizar la técnica de obtención de muestra con la finalidad de aumentar su rendimiento y establecer criterios de cómo interpretar un hemocultivo positivo.

\section{Referencias bibliográficas}

1.- Stoll B J, Hansen N, Fanaroff A A, Wright L L, Carlo W A, Ehrenkranz RA, et al. Late-onset sepsis in very low birth weight neonates: the experience of the NICHD Neonatal Research Network. Pediatrics 2002; 110: 285-91.

2.- Stoll B J, Hansen N I, Bell E F, Shankaran S, Laptook A R, Walsh M C,et al. Neonatal outcomes of extremely preterm infants from the NICHD Neonatal Research Network. Pediatrics 2010; 126(3): 443-56. DOI: 10.1542/ peds.2009-2959.

3.- Díaz M, Trigoso Agudo C, Damiani Moisés E, Ode Hiramatsu Y, Navía Bueno M. Agentes infecciosos en sepsis neonatal servicio de neonatología Hospital de la mujer-Inlasa. Cuad Hosp Clin 2005; 50 (1): 9-15.

4.- Genes L, Lacarruba J, Mir R, Céspedes E,
Mendieta E. Sepsis neonatal. Caracterización de recién nacidos de muy bajo peso. Experiencia once años. Pediatr (Asunción) 2013; 40 (2): 145-54.

5.- Tapia J L, Reichhard C, Saldías M I, Abarzúa F, Pérez M E, González A, et al. Sepsis neonatal en la era de la profilaxis antimicrobiana prenatal. Rev Chil Infectol 2007; 24 (2): 111-6. http:// dx.doi.org/10.4067/S0716-10182007000200004

6.- Cohen-Wolkowiez M, Moran C, 
Benjamin D K, Cotten C M, Clark R H, Benjamin D K Jr, et al. Early and late onset sepsis in late preterm infants. Pediatr Infect Dis J 2009; 28 (12): 1052-6.

7.- Rubin L, Sánchez P, Siegel J, Levine G, Saiman L, Jarvis W. Evaluation and treatment of neonates with suspected late-onset sepsis: a survey of neonatologists' practices. Pediatrics 2002; 110 (4): e42.

8.- Norberg A, Christopher N, Ramundo M, Bower J, Berman S. Contamination rates of blood cultures obtained by dedicated phlebotomy vs intravenous catheter. JAMA 2003; 289 (6): 726-9.

9.- Nizet V, Klein J O. Bacterial sepsis and meningitis. In: Infectious diseases of the Fetus and Newborn Infant, 8th ed, Remington JS, et al (Eds), Elsevier Saunders, Philadelphia 2016. p. 217.

10.- Bard J, McElvania TeKippe E. Diagnosis of bloodstream infections in children. J Clin Microbiol 2016; 54: 1418-24. doi: 10.1128/ JCM.02919-15. Epub 2016 Jan 27

11.- Clinical and Laboratory Standards Institute (CLSI). Principles and Procedures for Blood Cultures; Approved Guideline. CLSI document M47-A. Wayne, PA: Clinical and Laboratory Standards Institute 2007.

12.- Creger R, Weeman K, Jacobs M, Morrissey A, Parker P, Fox R, et al. Lack of utility of the lysis-centrifugation blood culture method for detection of fungemia in immunocompromised cancer patients. J Clin Microbiol 1998; 36 (1): 290-3.

13.- Petti A, Zaidi K, Mirrett S, Reller L. Comparison of Isolator 1.5 and BACTEC NR660 aerobic 6A blood culture systems for detection of fungemia in children. J Clin Microbiol 1996; 34 (8): 1877-9.

14.- García P, Pérez C. Hemocultivos: clasificación, indicaciones e interpretación de sus resultados en el diagnóstico de bacteriemia. Rev Chilena Infectol 1997; 14 (3): 177-88.

15.- Dietzman D, Fischer G, Schoenknetcht F. Neonatal Escherichia coli septicemia-bacterial counts in blood. J Pediatric 1974; 85 (1): 12830.

16.- Schelonka R, Chai M, Yoder B, Hensley D, Brockett R, Ascher D. Volume of blood required to detect common neonatal pathogens. J Pediatr 1996; 129(2): 275-8.

17.- Kellog J, Ferrentino F, Goodstein M, Liss J, Shapiro S, Bankert D. Frequency of low level bacteriemia in infants from birth to two months of age. Pediatr Infect Dis J 1997; 16 (4): 381-5.

18.- Hernandez-Bou S, Álvarez C, Campo Fernández M, García Herrero M, Gené A, Giménez Pérez, et al. Hemocultivos en urgencias pediátricas. Guía práctica de recomendaciones: indicaciones, técnica de extracción, procesamiento e interpretación. An Pediatr (Barc) 2016; 84(5): 294.el-9. DOI: 10.1016/j.anpede.2015.06.020

19.- Saito T, Sensda K, Takakura S, Fujihara N, Kudo T, Linuma Y, et al. Anaerobic bacteremia: the yield of positive anaerobic blood cultures: patients characteristics and potential risk factors. Clin Chem Lab Med 2003; 41: 293-7. DOI: 10.1515/CCLM.2003.046

20.- H1 Zaidi A, Knaut A, Mirrett S, Reller L. Value of routine anaerobic blood cultures for pediatric patients. J Pediatr 1995; 127: 263-8.

21.- Ruiz-Giardin J, Noguerado A, DelgadoIribarren A, Valverde-Canovas J, ArandaCosgaya A, Delgado-Yague $\mathrm{M}$ et al. Modelo clínico predictivo y validación de bacteriemias por anaerobios (incluidas las bacteriemias polimicrobianas). Enferm Infecc Microbiol Clin 2010: 28: 421-9. DOI: 10.1016/j. eimc.2009.09.011

22.- Brook I. Bacteriemia due to anaerobic bacteria in newborns. J Perinatol 1990; 10: 351-6.

23.- Messbarger N, Neemann K. Role of anaerobic blood cultures in neonatal bacteremia. J Pediatr Infect Dis Soc 2017. doi: 10.1093/jpids/pix088. En prensa.

24.- Yaacobi N, Bar-Meir M, Shchors I, Bromiker R. A prospective controlled trial of the optimal volume for neonatal blood cultures. Pediatr Infect Dis J 2015; 34 (4): 351-4. DOI: 10.1097/ INF.0000000000000594

25.- Sakar S, Bhagat I, DeCristofaro J, Wiswell T, Spitzer A. A study of the role of multiple site blood cultures in the evaluation of neonatal sepsis. J Perinatol 2006; 26: 18-22. DOI: 10.1038/sj.jp.7211410

26.- Acuña M, O’Ryan M, Cofré J, Alvarez I, Benadof D, Rodríguez P, et al. Differential time to positivity and quantitative cultures for non invasive diagnosis of catheter related blood stream infection in children. Pediatr Infect Dis J 2008; 27 (8): 681-5. doi: 10.1097/ INF.0b013e31816d1 e00.

27.- Gaur A, Flynn P, Heine D. Diagnosis of catheter-related bloodstream infections among pediatric oncology patients lacking a peripheral culture, using differential time to detection. Pediatr Infect Dis J 2005;24 (5): 445-9.

28.- Franklin J, Gaur A, Shenep J, Hu X J, Flynn $P$ M. In situ diagnosis of central venous catheter-related bloodstream infection without peripheral blood culture. Pediatr Infect Dis J 2004; 23: 614-8.

29.- Wolf, J, Curtis, N, Worth, LJ, Flynn, PM. Central line-associated bloodstream infection in children: an update on treatment. Pediatr Infect Dis J 2013; 32 (8): 905-10. doi: 10.1097/ INF.0b013e3182996b6e

30.- Pourcyrous M, Korones S, Bada H, Patterson T, Baselski V. Indwelling umbilical arterial catheter: a preferred sampling site for blood culture. Pediatrics 1988; 81 (6): 821-5

31.- Anagnostakis D, Kamba A, Petrochilou V, Arseni A, Matsaniotis N. Risk of infection associated with umbilical vein catheterization. A prospective study in 75 newborn infants. $\mathrm{J}$ Pediatr 1975; 86 (5): 759-65.

32.- Polin J, Knox I, Baumgart S, Campman E, Mennuti M, Polin R. Use of umbilical cord blood culture for detection of neonatal bacteremia. Obstet Gynecol 1981; 57 (2): 2337.

33.- Carroll P D, Christensen R D. New and underutilized uses of umbilical cord blood in neonatal care. Matem Health Neonatol Perinatol 2015; 1(1): 16. doi: 10.1186/s40748015-0017-2. eCollection 2015.

34.- Meena J, Charles M, Kali A, Ramakrishnan S, Gosh S, Seetha K. Utility of cord blood culture in early onset neonatal sepsis. Australas Med J 2015; 8 (8): 263-7. http://dx.doi.org/10.4066/ AMJ.2015.2460

35.- Beeram MR, Loughran C, Cipriani C, Govande V. Utilization of umbilical cord blood for the evaluation of group B streptococcal sepsis screening. Clin Pediatr [Phila]. 2012; 51(5): 447-53. doi: 10.1177/0009922811431882. Epub 2011 Dec 22

36.- Kalathia M, Shingala P, Parmar P, Parikh Y, Kalathia I. Study of umbilical cord blood culture in diagnosis of early-onset sepsis among newborns with high-risk factors. J Clin Neonatol 2013; 2 (4): 169-72. doi: 10.4103/2249-4847.123092.

37.- Polin R A. Management of neonates with suspected or proven early-onset bacterial sepsis. Pediatrics [Internet]. 2012; 129(5): 1006-15. Available from: http://pediatrics. aappublications.org/cgi/doi/10.1542/peds.20120541

38.- Diomedi A, Chacón E, Delpiano L, Hervé B, Jemenao M, Medel M, et al. Antisépticos y desinfectantes: apuntando al uso racional. Recomendaciones del Comité Consultivo de Infecciones Asociadas a la Atención de Salud, Sociedad Chilena de Infectología. Rev Chilena Infectol 2017; 34 (2): 156-74. http://dx.doi. org/10.4067/S0716-10182017000200010

39.- Nuntnarumit P, Sangsuksawang N. A Randomized controlled trial of $1 \%$ aqueous chlorhexidine gluconate compared with $10 \%$ povidone-iodine for topical antiseptic in neonates effects on blood culture contamination rates. Infect Control Hosp Epidemiology 2013; 34: 430-2. doi: 10.1086/669863. Epub 2013 Feb 14.

40.- Abdelhamid S. Time to positivity and antibiotic sensitivity of neonatal blood cultures. J Glob Infect Dis 2017; 9 (3): 102-7. doi: 10.4103/jgid. jgid_1_17 